\section{Bello M \\ Garandawa H \\ Mustapha Z \\ Isa A \\ Tahir C \\ Ngamdu YB \\ Machoko Y

Abubakar H
Djossi SK

\title{
Frontonasal dysplasia Sequence : A case report
}

DOI:http://dx.doi.org/10.4314/njp.v41i2,17

Accepted: 3rd January 2014

Bello M ( )

Machoko Y, Abubakar H, Djossi SK

Department of Pediatrics,

Garandawa H, Isa A, Ngamdu YB

Department of ENT Surgery,

Mustapha Z

Department of Radiology,

Tahir C

Burns and Plastic Unit,

Department of Surgery,

University of Maiduguri Teaching

Hospital.

Maiduguri, Borno State, Nigeria

\begin{abstract}
Frontonasal dysplasia (FND) is a very rare congenital abnormality in which the mid face does not develop normally. It affects mainly the head and face. Cause is unknown but may be sporadic or familial. We report a rare case of a full term baby who
\end{abstract}

presented with classical features of FND in Maiduguri, Nigeria. Management difficulty in resource limited setting is highlighted.

Key words: Dysmorphism, Frontonasal dysplasia, Neonate.

\section{Introduction}

Frontonasal dysplasia (FND) sequence also called median cleft face syndrome is a rare condition in which the face does not develop normally with only a few reported cases worldwide and none from Nigeria ${ }^{1,2}$. The cause of this disorder is unknown, with a sporadic occurrence, it may occasionally be familial ${ }^{3}$. FND affects the head and face mainly but occasional abnormalities are found in the eyes, the brain and heart ${ }^{4}$. We report here a case of FND with agenesis of Corpus callosum, a usual finding in this disorder.

\section{Case Report}

Six hours old, term male neonate was brought into the Special Care Baby Unit (SCBU) of the University of Maiduguri Teaching Hospital on account of dysmorphic facie. Baby was delivered to a 27 year old Para 2 woman through spontaneous vertex delivery at home assisted by traditional birth attendant. Parents were not consanguineously married. Mother had antenatal care at a primary health care facility, registered at two months gestation. She was said to have had fever during the first month of gestation associated with abdominal pain for which she had paracetamol and B-complex given orally. No significant history of ingestion of herbal preparations. The patient was the $2^{\text {nd }}$ child in a monogamous setting; mother is unemployed while the father was a security officer. No history of similar features in the other sib. Examination at presentation revealed length of $50 \mathrm{~cm}$, occipito-frontal circumference of $33 \mathrm{~cm}$ and weight of $3.05 \mathrm{~kg}$. The baby has obvious facial deformity (Fig 1), widow's peak and cranium bifidum occultum on forehead, in respiratory distress, with divided nostrils, bifid nose and serous discharge with low set ears.

\section{Fig 1: Showing facial} deformity, low set ears with deformity of the nose and widow's peak

Fig 2: Showing: Widow's Peak, deformed nose with widely spaced eyes and serous discharge from the nostrils
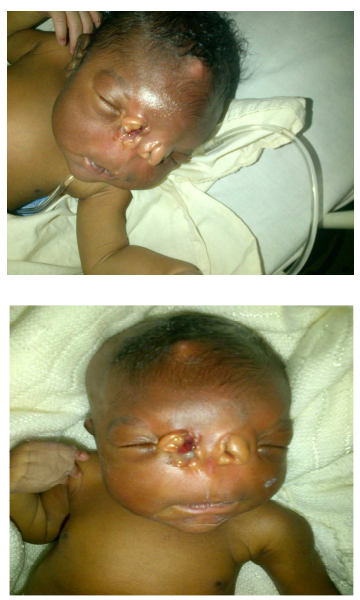
Investigations done reveals: $\mathrm{FBC}-\mathrm{PCV}=50 \%, \mathrm{WBC}=$ $7.8 \times 10^{9} / \mathrm{L}, \mathrm{N}=58 \%, \mathrm{~L}=34 \%, \mathrm{E}=4 \%, \mathrm{M}=4 \%$, Blood Culture - No growth. CT-Scan: The 3D reformation shows absent nasal bones with hypoplasia and lack of midline fusion of the frontal bones which were deficient bilaterally. Corpus callosum agenesis is noted. The cerebral and cerebellar cortices are normal. The right anterior horn of the lateral ventricle appears slightly narrowed? Positional, the brain stem is normal; features are in keeping with medium cleft face syndrome (Fig. 3)

Fig 3: CT Brain - 3 D Reformated image showing sutural diastesis and defect in frontal bone

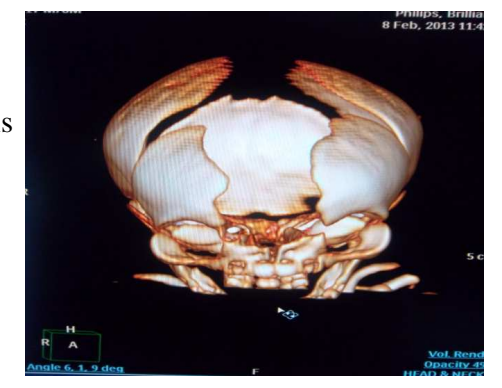

Fig 4: CT Brain sagittal image showing agenesis of corpus callosum

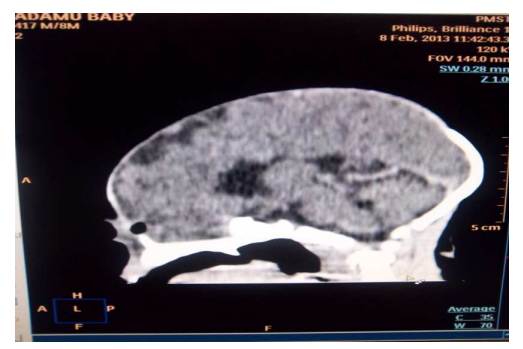

Fig 5: CT Brain - Axial Image - showing absence of frontal horn of lateral ventricles

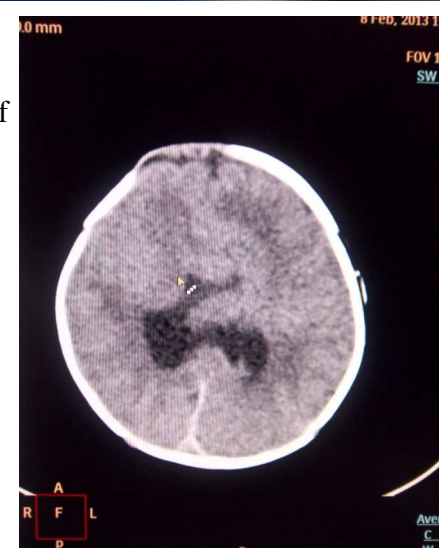

Baby had an oropharyngeal airway was put in place and nasogastric tube size 6 FR passed for feeding with expressed breastmilk. Problems encountered were: nasal cavity not patent posteriorly and respiratory difficulty on removal of oropharyngeal airway. Baby was planned for an initial posterior choanoraphy so as to enable nasal breathing without interfering with feeding and later cosmetic surgery, but baby's parents refused medical advice and took baby home against medical advice at the age of 18 days.

\section{Discussion}

DeMeyer first described the transitional gradations in severity of this presumed single primary localized defect in 33 cases and called this pattern of anomaly the median cleft face syndrome ${ }^{1}$. Sedano and colleaques subse- quently extended these observations and recommended frontonasal dysplasia as a more appropriate designation for this defect.

Frontonasal dysplasia (FND) is a rare developmental defect of craniofacial region where the midface does not develop normally ${ }^{1,2,4}$. The exact cause of FND is unknown. Anomalies can be explained by single malformation, though most cases are sporadic. It has also been suggested that a defect involving chromosomes 3q23, $3 q 27,7 q 21$ and $11 q 21$ might play a role ${ }^{2}, 3$. In our case, chromosomal studies were not done due to absence of facilities. The parents of an affected child can expect the risk to be $25 \%$ for the next child. ${ }^{5,6}$

The embryogenic origin of FND is in the period prior to the $28-\mathrm{mm}$ crown - rump length stages. During the $3^{\text {rd }}$ week of gestation, two areas of thickened ectoderm, the olfactory areas appear immediately under the forebrain in the anterior wall of the stomodeum. By the up growth of the surrounding parts, these areas are converted into pits, the olfactory pits, which indent the frontonasal prominence and divide into a medial and two lateral nasal processes. Frontonasal dysplasia is due to deficient remodeling of the nasal capsule, which causes the future fronto-naso-ethmoidal complex to freeze in the fetal form ${ }^{3}$.

There may be few defects in the milder form of FND where as the severe defects affects the eyes (hypertelorism, lateral displacement), forehead (widow's peak, deficit in midline frontal bone - cranium bifidum occultum), and the nose - (variably affected from notched broad nasal tip to completely divided nostrils with hypoplasia to absence of the prolabium and premaxilla with a median cleft lip) ${ }^{7,8}$ - as was the case in our patient. Our patient did not however have some features such as absence of prolabium and pre-maxilla and median cleft.

As neonates are nasal breathers, simultaneous sucking and breathing leads to respiratory distress ${ }^{9}$. This was the case in our patient at the time they presented to us. Oral airway and orogastric feeding was instituted temporarily, canalization of the nasal passage was planned for to allow breastfeeding and breathing with cosmetic surgery planned for a later time but parents refused and took baby home against medical advice as reported in other series $^{9}$. Despite efforts at getting the hospital to waive charges off for admission and surgery, the parents decline staying in hospital for further medical management on grounds of father going on posting outside the country. Further follow-up was also not possible in this instance as both mother and baby travel out of Maiduguri to their home town immediately after discharge.

Individuals with FND tend to have normal lifespan with average intelligence. Affected individuals with severe form of the disorder may die shortly after birth if corrective surgery to establish normal feeding and breathing is not performed as soon as possible. 


\section{Conclusion}

In conclusion, FND is a very rare disorder; the aetiology is unknown and may require a multidisciplinary management approach. Lack of adequate diagnostic facilities, Socio-economic and cultural factors may be a problem in our own setting. We suggest making services available free of charge to under fives and particularly the newborn, this will have helped in prompt and effective management of such babies, thereby reducing neonatal morbidity and mortality.

\section{References}

1. Jones KL. "Frontonasal Dysplasia Sequence" In Smith's Recognizable Pattern of Human Malformation, sixth Edition, Elsevier Saunders, 2006; 268-269.

2. Seema Sharma, Vipin Sharma, Meenakshi Bothra. Frontonasal dysplasia. J Neurosc Rural Pract 2012; 3:65-67.

3. Median Facial Cleft Syndrome. http://www.mypacs.net/cases/ median-facial-cleft-syndrome
4. Handbook of Genetic Counseling/Frontonasal DysplasiaMedian Cleft Face Syndrome. http://en.wikibooks.org/wiki/

Hand-

book_of_Genetic_Counselling/ Frontonasal Dysplasia-Median Cleft Face Syndrome.

5. Fox JW, Golden GT, Edgerton MT. Frontonasal dysplasia with alar clefts in two sisters. Genetic considerations and surgical correction. Plast Reconstr Surg 1976; 57:553-6

6. Lorenz P, Prager B, Tellkamp H. Frontonasal dysplasia: Case report and review of literature. Kinderarzt Prax 1990; 58:415-20
7. Gorlin RJ, Cohen MM, and Levin SL. "Syndromes with Unusual Facies." Syndromes of the Head and Neck. Oxford University Press (1990): 785-788

8. Apere H, Serville F, Collet B, Billeaud C, Sandler B. Frontonasal dysplasia or the median cleft face syndrome: a case report. J Genet Hum. 1989; 37(4-5):431-6.

9. Debabrata Das, Gobinda Das, Sibnath Gayen, Arpita Konar. Median facial cleft in amniotic band syndrome. Meajo, 2011, 18 : 192-194. 